\title{
Natural and Man-Made Chemicals in North American Soils-Continental-Scale Pilot Study Completed
}

The U.S. Geological Survey and the Geological Survey of Canada recently completed a continental-scale pilot study for a proposed geochemical survey of North American soils. This survey will provide baseline soil chemistry data against which future changes in soil composition can be measured and that can be used by Federal, State/Provincial, and local agencies when making riskassessment and land-use decisions. Agencies that will use these data include

- U.S. Department of the Interior

- Natural Resources Canada

- U.S. Environmental Protection Agency

- Environment Canada

- Centers for Disease Control and Prevention

- Health Canada

- State, Provincial, and local departments of environmental protection and health departments

\section{Introduction}

Soil is a critical natural resource that plays a key role in determining human health and ecosystem integrity, supporting food production and the natural recycling of carbon and essential nutrients in the environment. Soil also stores water used by plants in dry seasons. On the other hand, many communities dispose of solid and liquid wastes from households and from agricultural and industrial processes by dumping them onto the soil. Although soil is so important in our everyday lives, our knowledge of the concentration and distribution of naturally occurring and man-made chemicals in the soils of North America is very limited. As a result, establishing standards for soil clean-up levels, evaluating the effects on human health of chronic exposure to contaminated soils, and determining the impact of new land-use practices on the environment are extremely difficult without baseline data for comparison.

In order to improve our understanding of the types of chemicals and elements that are normally found in soil and the location and causes of elevated or depleted levels, the U.S. Geological Survey (USGS), the Geological Survey of Canada (GSC), and the Mexican Geological Survey (Servicio Geológico Mexicano, or SGM) are collaborating on the North American Soil Geochemical Landscapes Survey, a project to survey the chemical composition of soils over all of North America. The goal of this project is to map the concentrations and spatial distribution of elements and selected chemicals across North America.
Such a geochemical survey could be used by Federal, State/Provincial, and local agencies as a baseline for risk-based assessments of contaminated land and in determining the impact of land-use decisions (such as timber harvests, mining, industrial activities, or landfill permits) on the soil, the environment, and human health. The survey could also be used as a baseline in writing environmental impact statements for new projects that may affect our environment or to monitor the effect of ongoing activities on soil composition in surrounding communities. The survey is intended to replace older, outdated, and incomplete data that are currently being used by land-management, regulatory, and public health agencies.

\section{Project Status}

The North American Soil Geochemical Landscapes Survey was officially launched in 2003 at a workshop attended by 112 representatives from more than 40 North American governmental agencies, academic institutions, environmental consultants, and the medical community. At the workshop, recommendations regarding study design, sampling procedures, and analytical methods for the continental-scale survey were adopted. Overall, it is estimated that more than 40,000 soil samples will be collected and tested for more than 40 elements and chemical compounds during the multiyear project.

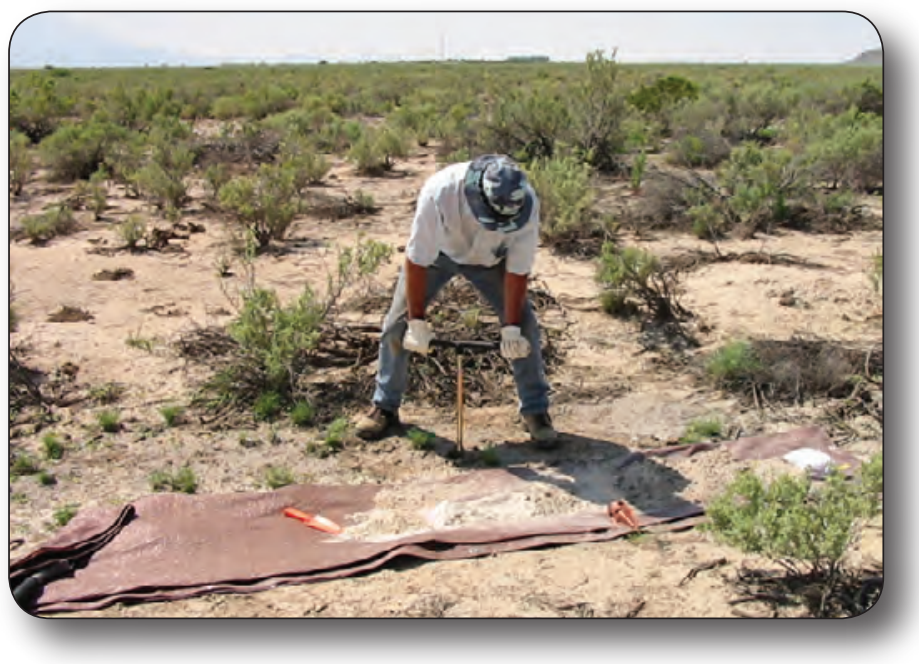

A USGS scientist collecting soil samples in southern New Mexico. Samples were collected approximately every $40 \mathrm{~km}$ along two transects. 
In order to test the recommendations adopted at the workshop, a pilot study was initiated in 2004 to

- test and refine the recommended procedures,

- optimize the logistics for carrying out a continental-scale study,

- assess the relationships among any observed patterns in soil composition and possible human and natural contributions to soil composition, including the types of rocks and other geologic features, and

- investigate the feasibility of mapping the microbial characteristics of soil on such a large scale.

For the pilot study, samples were collected along two lines, or transects. One transect ran north to south from northern Manitoba, Canada, to the United States-Mexico border. In 2006, sampling along this transect is planned to extend into Mexico. The other transect follows the 38th parallel in the United States from east to west, beginning in Maryland and ending just north of San Francisco, Calif. These transects cross many types of soil and rock, land used in various ways, and ecological boundaries, allowing for rigorous testing of sampling methods over a broad range of conditions.

\section{Preliminary Results}

Preliminary results of the chemical analyses of the soils collected in the pilot study show systematic continental-scale patterns of element concentrations. These patterns can be correlated with the composition of the underlying bedrock and glacial deposits (soil parent material), and with climate-related processes that affect vegetation cover, soil moisture content, and levels of organic matter in the soil.

For example, the geochemical map to the right shows the concentration of mercury in soils along the east-west transect. Note that mercury concentrations along the eastern half of the transect are higher than along the western half of the transect. Mercury tends to bind strongly to organic matter in soil, so this variation along the transect is consistent with higher levels of rainfall in the Eastern United States and consequent higher overall organic content of the soils than in the Western United States. The pilot study data also show that mercury levels in soils in Manitoba, Canada, are generally lower than in the Eastern United States, although the organic contents are very similar. This difference could be explained by the presence of more coal-fired power plants and heavy industrial sites, which may emit mercury into the atmosphere, in the Eastern United States than in Manitoba. Atmospheric mercury has been shown to be deposited onto surrounding soils by rain and snowfall, where it binds to the organic matter in the soil.

These data will help environmental scientists, geologists, ecologists, and other scientists study the effects of our activities on our environment. Information from this survey will also be useful to those managing our natural resources for determining what land uses may or may not be appropriate for certain areas and for studying the effects new activities might have on the environment and on human health.

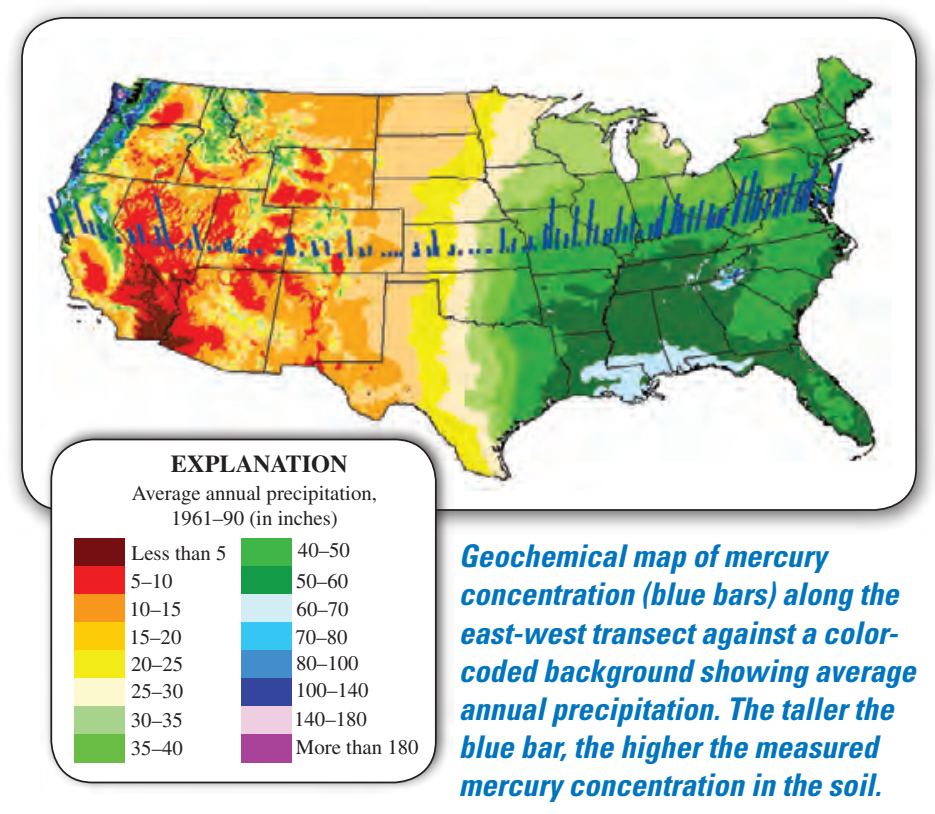

Next Steps

The pilot study to test sample design, sampling protocols, and analytical methods for the North American Soil Geochemical Landscapes Survey has been successfully completed. With the completion of this important milestone, protocols for final sampling and analytical methods will be finalized in 2006, and sampling for the full study will begin in 2007.

\section{Summary}

Our ability to understand the impact of soil composition on human health is rapidly progressing through research that is being conducted around the world. The North American Soil Geochemical Landscapes Survey will help us better understand the current trends in soil composition and compare them with future changes resulting from natural events, such as hurricanes, dust storms, wildfires, and volcanic eruptions, or from manmade causes, such as contamination by industrial, agricultural, or urban sources. The data from this new survey will supersede the fragmented and outdated chemical and biological survey data that currently exist for small parts of North America and will be available to all researchers concerned with environmental monitoring and with effects of soil composition on human and animal health.

For more information about the USGS or GSC work on the North American Soil Geochemical Landscapes Survey:

- David B. Smith, U.S. Geological Survey, dsmith@usgs.gov

- Martin B. Goldhaber, U.S. Geological Survey, mgold@usgs.gov

- Andy Rencz, Geological Survey of Canada, rencz@NRCan.gc.ca

- Robert G. Garrett, Geological Survey of Canada, garrett@NRCan.gc.ca 